\title{
On the Spirit of "Dialogue" in Literary Criticism
}

\author{
Ying Chen \\ College of Literature, Dalian University, Dalian, China \\ Email: clariey1102@163.com
}

How to cite this paper: Chen, Y. (2018). On the Spirit of "Dialogue" in Literary Criticism. Advances in Literary Study, 6, 142-152.

https://doi.org/10.4236/als.2018.63012

Received: July 3, 2018

Accepted: July 21, 2018

Published: July 24, 2018

Copyright $\odot 2018$ by author and Scientific Research Publishing Inc. This work is licensed under the Creative Commons Attribution International License (CC BY 4.0).

http://creativecommons.org/licenses/by/4.0/

\section{(c) (i) Open Access}

\begin{abstract}
Today's literary criticism has reached a deadlock between dogmatic theory criticism and theory of immanence criticism, and lacks a spirit of "dialogue". This kind of "dialogue" is not to obliterate the discourse right of other literature, but a kind of "harmony but difference", which is the balance of subjectivity. It is not the rigid binding of the local experience that is cut one-sidedly by western experience, but a kind of "not holding a corner", which is explained in two directions and equally. It is not the stiff patchwork of various theoretical discourses, but the fusion of horizons based on inherent logic and identity.
\end{abstract}

\section{Keywords}

Literary Criticism, Dialogue, Harmony but Difference, Not Holding a Corner

\section{Introduction}

Nowadays, literary criticism is more and more deadlocked between dogmatic theory criticism and theory of immanence criticism. The former evaluates the writer's works with the established monistic thought, guides the writer's creation with the attitude of "looking down", and only allows the voice of the critic to exist, thus developing into the critic's "discourse monologue". The latter focuses on exploring the inner details of literary works, and leads the literary research to the inherent "immanence" of the works, thus causing the critics to be speechless. In order to break the rigid and closed critical situation, we may introduce the "dialogue" spirit hidden in the Chinese and Western cultural traditions into the field of literary criticism, and construct a pluralistic dialogue relationship between critics and writers, critics and critics, critics and readers, critics and works, and so on. Then, in the reflection of the same thoughts or the confrontation of different thoughts, it results in a unique literary insight. This kind of "dialogue" is not to obliterate the discourse right of other literature, but a kind of "harmony 
but difference", which is the balance of subjectivity. It is not the rigid binding of the local experience that is cut one-sidedly by western experience, but a kind of "not holding a corner", which is explained in two directions and equally. It is not the stiff patchwork of various theoretical discourses, but the fusion of horizons based on inherent logic and identity.

It can be said that the spirit of "dialogue" has become an urgent demand of contemporary literary criticism, which is of great significance to overcome the existing difficulties of criticism and to construct the contemporary literary criticism theory. This paper discusses the cultural origin, the theoretical foundation, the core spirit and the logic of thought in an in-depth manner.

\section{The Cultural Origin of "Dialogue"}

Chinese culture has always attached importance to the exchange and integration of ideas between different subjects, and the thought of dialogue has a long history. Tao, as the central category of ancient philosophy, declares the spirit of "dialogue" from the two aspects of society and nature. The Confucianists in the pre-Qin Dynasty cut into the Tao from the ethical orientation and emphasized humanism. No matter Confucius's "The man of humanity wishing to be successful himself, seeks to help others to be winner; wishing to develop himself, seeks to help others to develop. To restrain oneself and abide by rites is humanity", or Mencius's "Those who love others love them, and those who respect others respect them", they all emphasize the deconstruction of self-centeredism, trying to eliminate the opposition between people and self, and establish unity of others and oneself. The purpose and mission of the theory is to establish the "dialogue" mode, which emphasizes communication and communication. Different from Confucian ethics, Taoists in the pre-Qin Dynasty cut into the concept of Tao from the cosmological orientation and put forward the Tao of Heaven. Both Laozi's "Tao can be Tao, extraordinary Tao" and Chuang Tzu's "virtue and virtue in Tao, Tao in heaven" all turn "Tao" into a universal law closely related to everything in heaven and earth, and strive to seek dialogue between heaven and man and unity between heaven and man. striving to seek the dialogue between heaven and man and the unity of nature and man, and trying to eliminate all kinds of solid and firm beliefs in the wider universe, and to move towards a kind of complete "unity doctrine".

Taoism's so-called "The Tao of Heaven" and Confucianism's so-called "The Tao of humanity" are quite different in cultural standpoint. The former has the internal dialectical self-deconstruction structure, while the latter has the main body's external deconstruction mode. However, as far as the fundamental philosophical orientation is concerned, the two are in fact same inherently, both in order to avoid the extremes of a bigotry, to adhere to the openness of human communication and to seek creative and transcendental spiritual communication.

If Tao is the aim of Chinese dialogue, harmony is exactly the embodiment of 
its inner principle. The original meaning of the word "harmony" refers to the corresponding sound to sound of singing, while the latter refers to the consistent or harmonious relationship between different things. Its core connotation is to discuss how many different factors coexist in different relational networks from different fields, and to achieve new harmony and unity by coordinating different factors. In Chinese traditional culture, whether the Confucianism that pays attention to the relationship between man and man, or the Taoism that pays attention to the relationship between man and nature, both embody the spirit of "harmony" which respects the difference, coexists equally, and harmoniously unifies in essence. For example, Chuang-tzu's highest ideal is "Taihe and all things", who hope the world can achieve the most perfect harmony. Confucius advocated "harmony but difference", and believed that different thought subjects should neither attach themselves to others, nor should they hold one end of each other, but should form new ideas in the exchange and complementation of each other.

As one of the core concepts of Chinese traditional culture, "harmony" emphasizes respect for self and others, openness and tolerance of thought, harmony and unity between man and nature. It integrates Confucian ethics with Taoist cosmology, generalizes the principle of intercommunion embodied in benevolence into universal truth of the universe, and "humanity" is also promoted as an omnipresent way of the universe, with an open mind that dispels the subject-object treatment, dispels all kinds of inflexible relativism, becoming a generalized "Tao" that is truly impassable and unreachable.

The spirit of dialogue in Chinese traditional culture has left a deep impression in the classical literary theory. In the Analects of Confucius, Zihan, there is a word "I can deduce both ends", which means wandering between ends to search for the most appropriate answer to a question. The speculative judgment of traditional literary theory in our country has the characteristics of "clasping its two ends" and not holding a corner. Whether it is the "law but wrong" in the creative theory, the "natural nature" in the theory of style, the beauty of "good manners" in the theory of style, the beauty of "neutralization", or the "unvintage" and "common change" of the theory of development, all roam between two opposing categories, theories or ideas. Not sticking to either side in the bipolar dialogue, but striving to find the right "degree" to embody the power of balance and the beauty of harmony, and to respect differences, explore the way of understanding under the control of the spirit of free and equal dialogue. This way of thinking not only dispels the bigotry of theoretical understanding, but also opens up an innovative and open speculative space. Its contents are complex and profound, which cannot be compared with any kind of monistic or dual epistemology.

In the West, the idea of dialogue has a long history. As early as ancient Greece, the art of conversation existed in the field of philosophy, focusing on the exchange and debate of ideas. Because of the limitation of the subject's ability of self-consciousness and reflection at that time, in the dialogue before Socrates, 
there was not a general category of arguments between the two sides of the dialogue as the basic point of the dialogue, which resulted in the dialogue becoming a tool for the debater to preach his own point of view, or even sophistry. In order to avoid the dialogue becoming the monologue of the speaker to preach his ideas, Socrates put forward the general category of "idea" as the basic reference of the dialogue, to stimulate the opponent to explore the truth conclusion. In his view, dialogue is the "midwife" and "matchmaker" of truth, and an effective means for human beings to explore truth and knowledge. Any dialogue subject is equal to the idea and cannot be regarded as the holder of truth. This is because the idea itself, as the aim of dialogue, is uncertain and vague. It is merely "seeking difference in 'chaos' and seeking rules in 'change' so that knowledge has a stable and reliable foundation" (Ye, 1986: p. 173). Socrates' dialogue is an endless inquiry into truth between subjects, and a vivid and rich exchange of ideas. It respects differences, emphasizes equality, and embodies the spirit of dialogue in the modern sense.

In many theories of Socrates' and western modern dialogist philosophy, we have found the echo of the ancient Chinese dialogue philosophy's voice which has been silent for a long time. It can be said that the "dialogue", conceived in different cultural contexts of China and the West, has a common intellectual search spirit of "thinking in one".

Chinese dialogue emphasizes "Tao", and Socrates dialogue focuses on "reason". Both of them are intellectual questions about existence of things. "Tao" and "Li" have the consistency of the core, the two are not an entity of the concept, cannot be determined, inexplicable, drifting nihilism, there is no clear definition can cover, but at the same time, both include the rules, principles, the universe and other central meaning. Either "idea" or "Tao" is the perfect unity of thought and existence, which always exists in the world of representation, and does not need to be found at the bottom of the representation, let alone to analyze and disassemble it. Therefore, whether Socrates' dialogue seeking "principle" or the Chinese dialogue "Tao", both respect phenomena, feeling, life, are in a constantly growing and changing universe to explore the reality of a certain appearance, and both in line with the "thought in one" research consciousness, explore the infinite growth of the meaning of space.

\section{The Theoretical Basis of "Dialogue"}

The confluence of the thought of dialogue between China and the West is not an accidental phenomenon in the development of culture, but is based on the theory of dialogism, on the Chinese and Western isomorphism, and on the open thinking that transcends the dualistic opposites.

Metaphysics, as the basis of Western cultural tradition, is a dominant philosophy in western modern times. It divides the whole world into opposite poles: phenomena and nature. This kind of mechanical duality is helpful to mankind in the budding period of civilization. It gives a superficial interpretability to the 
chaotic reality experienced by people. By asking and answering questions about the nature of all kinds of things, by asking about the origin and causes of things, and by expelling all kinds of worries, transforming chaos into order, conflict into harmony, and thus constructing a rational and clear understanding of the world. However, as metaphysics goes to extremes, as a synonym for human reason, it finds unity in a wide variety of diversity, abstracts intangible concepts in rapidly changing phenomena, and finds a single invisible reality behind the visible, pluralistic appearance of the world. It becomes what Heraclitus calls the "logos" and derives from it a series of dualistic thinking patterns such as spirit/matter, other shore/shore, soul/body, essence/phenomenon, content/form, sensibility/rationality, affirmation/negation, reason/emotion, etc. This kind of dualistic thinking always seeks a kind of certainty and measurable truth beyond the appearance, obtains the knowledge through tracing the reason, transforms an abundant universe which is constantly growing and changing into a closed and unified drying system.

In the Chinese cultural tradition, the dialogical thinking of "clasping its two ends" and not holding a corner is obviously a powerful correction to metaphysical dualism. It never adheres to any category, theory or idea, but seeks to transcend the dialogue between the two poles, to break the closed and dreary state caused by all kinds of duality, and to pursue an open and free state of harmony. In the theory of question and answer logic and horizon blending in Gadamer's hermeneutics, and in Derrida's theory of subverting traditional metaphysical dualism with structuralism, in Bakhtin's theory of literary criticism "polyphonic" view, Habermas's "communicative rationality" theory, this way of thinking can be found a trace. They all show rebellion and transcendence to dualistic thinking mode in the spirit of respect for difference and tolerance and mutual exchange.

Of course, as a universal category, dialogue has a huge theoretical market, not only because of its open and innovative mode of thinking, but also based on its intrinsic spirit of human studies.

Dialogue itself implies the theoretical presupposition of human commonality. Regardless of race, colour, descent, age or location, they are what Mr. Qian Zhongshu calls "hairless bipods", with the inherent consistency of the form of human life, with similar regular behavior and continuity in the aspects of birth, old age, illness and death, sexual union, reproductive reproduction, and group structural ability. The basic forms of existence of life, like the common body, mind, senses and soul, make the experience of life and existence common, and naturally form the basic structure of meaning expression, such as life and death, love and hate, time and existence, joy and sadness, parting and meeting, hope and despair, etc. This makes the interpersonal dialogue have a common meaning structure paradigm. It is on the basis of this common human understanding that in the vision of the interlocutor, the self and the other can be placed in an equal relationship, and then in the exchange, the main meaning and value of both parties to the dialogue are recognized, and the equal state of each other is main- 
tained. Whether Confucius's "harmony but difference", "one wants to stand up, one wants to become a human being", or Heidegger's "the existence of man is a greeting and dialogue with the world", both of them establish the equal relationship of harmonious coexistence between human beings by dialogue, contain the equal consciousness of promoting the spirit of human studies, the democratic thought and the inquiry and interpretation of the ultimate meaning of human beings, and show the deep concern of thinkers for the existence of human beings.

\section{The Core Spirit of "Dialogue"}

In the history of Chinese literary criticism, literary theorists often make a comparative study of various literary phenomena or theoretical viewpoints in the form of fictional dialogue, and carry out dialogical criticism in the form of conversation or dialogue, resulting in the emergence of a large number of dialogue-based poetry, words, songs, comments and other critical styles. As a unique style of criticism, dialogical criticism is free, with realistic pertinence, practicability and flexibility.

However, what we call "dialogue" here does not mean the above-mentioned style of criticism, but a spirit of openness and tolerance with "a hundred schools of thought contend". As a metaphorical criticism spirit with broad connotation, "dialogue" involves every aspect of culture and every link of literature. According to the relationship between the subject of dialogue, it includes the network of communication between the author and the author, the author and the reader, the text and the text, and the communication between the text and the reader. According to the morphological characteristics of the dialogue, it can be divided into phenomenal connection, text interpretation, artistic evaluation, theoretical promotion and so on. According to the theme content of the dialogue, it includes literary ontology, creation law theory, text constitution theory, literary appreciation theory and so on. According to the level of dialogue, it has many meanings, such as spreading literary information, enriching the content of works, exploring the law of creation and opening up the space of thought. In short, dialogue is multidimensional, rich in content, difficult to be accurately summed up in one or two sentence. However, behind the connotation of such a beautiful "dialogue", we can still capture a core spirit that runs through all the time. This is the spirit of "harmony and difference" which emphasizes the subjectivity of each other and advocates the difference and equality.

The 13rd of the Analects of Confucius, Zilu, "the Master said, 'the superior man is friendly but different, and the villain is not at peace with each other.' Harmony but difference" means that the subject of thought neither adheres to others nor holds one end of each other in the discussion and contention, but forms new ideas in the exchange and complementation of each other. In the Historical Records of nations, Zheng Guo, "Harmony will produce real creatures, the same will not continue with the sum of others, so it can grow and ge- 
neralize; if it keeps the same, it will be abandoned... There will be no sound in one sound, there will be nothing in one culture, there will be no fruit in one taste, there will be no talk in one content". The meaning is that "the same", the pursuit is the absolute unity of things, it causes things to lose their own characteristics, and even no longer continue. If the sound is pure, there is no music, the taste is pure and there is no fruit. "Harmony" is different from "same". What it pursues is "seeing difference with others", which can make things flourish in the tension of difference and consistency. From this point of view, the so-called "harmony" is to seek an equal kind of interactive cognition and two-way complementation between the two parties with their own subjective dimensions, both in the same and in the differentiation of differences, but also in the differences, in order to achieve a mutual subjectivity of the balance. "Harmony without difference" is obviously not a simple homogenization or convergence between the two sides of the discourse, but a pluralistic complementarity of the principle of "always living in a disparity".

Literary criticism should not only recognize the independent existence of various theoretical discourses, respect their differences and subjectivity, but also seek communication and negotiation between different discourse in order to reach a certain standard and consensus. In essence, it is the idea of harmony but difference advocated by Confucianism. This kind of dialogue of "harmony but difference" is not the plane comparison of different words and times, but the omni-directional and three-dimensional "uniting" of human literary and artistic thought, showing the fusion of two dimensions: Historical relevance dimension (ancient and modern dialogue) and logical correlation dimension (Chinese and Western dialogue). On the one hand, critics should attach great importance to the connection between phenomena and theories, be good at tracing back to the source along the wave, and empirically trace the origin of various literary phenomena, literary ideas or creative schools. To find out the modern meaning of ancient literary theory, the modern academic field of vision and the ancient literary thoughts are exchanged, communicated and collided with each other, and then lead to the creative and contemporary sense of thought and theory. On the other hand, critics should also be good at melting Chinese and Western literary theories, They should not only face the Chinese literary tradition from the western perspective, but also examine the theoretical heritage of the West from the perspective of the Chinese and Western thoughts, and learn from each other through the shift style of mutual learning, so that from the mutual reflection of Chinese and Western thinking, profoundly elucidate each other through shifting mutual reference. In this way, in the broad space-time context, the historical relevance and the logical relevance are organically connected, sliding freely along the axis of time and space, which is neither subject to the time threshold of the old and new ideas, nor to the limitation of the Chinese and Western territories. Thus, it breaks through all kinds of boundaries and barriers, such as times, cultures and factions, and realizes the conference between ancient and present, be- 
tween China and the west.

It should be pointed out that the "dialogue" of criticism should not only be confined to the territory of literature and literary theory, but also means the communication and creation between different disciplines. Critics might as well use the "other's boundary" of other disciplines, such as classics, history, psychology, Zen, exegetics, and so on, to reflect on the laws of literature and art, so as to activate and renew the concepts or categories inherent in Chinese literary theory, and realize the effective grafting and transformation of the theory itself. Mr. Qian Zhongshu, for example, made a successful attempt in synaesthesia (Qian, 2002a: pp. 62-76). He came up with the famous sentence in Song Qi's "Yulou Spring", "Red apricot branch spring trouble". First of all, from the perspective of rhetoric, he analyzed the "theory of reason outside" used in the use of the word "noisy", and quoted Yan several ways, Mao Pang, Ma Yan, Huang Tingjian, and Chen Ye-yi from the perspective of rhetoric. Fan Chengda, Zhao Mengjian and other poets of different times have similar techniques of poetry. Later, he connected this poetic creation technique with the creation of different categories of painting theory, novel, prose and so on, which confirmed the universality of "feeling moving" in artistic creation. Then he cut into Liu Xie, Bai Juyi, Li Yishan, Lu Ji, Shi Kongtu, Shi Huihong, Shi Xiaoying, Shi Cangxue, as well as Aristotle, Homer, Ponte in the West from the angles of logic, literature, psychology, classics, Buddhism, and so on. Saint-Martin, Black, and other writers, critics, and disciples of Buddhism, who belong to different time and space and have different identities, making them carry on omni-directional and three-dimensional "dialogue" around the problem of "synaesthesia", and naturally endow "synaesthesia" with new theoretical connotation in the mutual proof and complementation of ideas from different subject fields.

From the point of view of ideological purport, the spirit of "dialogue" in literary criticism actually implies two potential value objectives: First, it is committed to the modern transformation of Chinese traditional literary theory, and explores how to open up the part of the traditional literary theory resources with life potential to modern, so that it can become an organic component and future growth basis of modern literary theory. The second is to establish or highlight the value and status of the Chinese literary tradition in the world literary theory in the intercultural dialogue, and to show the problems of human presence and encounter in literature through the "dialogue" to show the long accumulated literary experience and theoretical achievements in different cultural contexts and to solve the problems faced and encountered by mankind in literature.

\section{Thinking Logic of "Dialogue"}

Judging from the present situation of literary criticism, the spirit of "dialogue" is relatively lacking, or cannot reach a more conscious, larger and more extensive level. At present, there are three biased ways of criticism, one is the random echo under the cover of humanization and commercialization; One is the "cool re- 
view" made by the mass media and the irresponsibility of the entertainment "review of the times"; another is the abstract hypocrisy and stiff binding represented by the academic criticism. The three kinds of criticism have not established a deep spiritual "dialogue" with the works, writers and critics, nor have they reached the realm of "harmony but difference" from the perspective of "big culture". At present, if literary criticism wants to construct a new critical order, it must establish an open and compatible spirit of "dialogue" and a way of thinking. This "dialogue" is by no means a word spoken by the two sides, or the one side accompanying him, or a bar that has nothing to come from, but in the two-way equality of interaction to reach a common understanding of the "horizon fusion". Through the communication, confrontation, collision, absorption and confluence between the two sides, the valuable parts of the two sides can be fully revealed, and the real discoveries and achievements can be made, and finally the theoretical logic will be self-consistent.

On the general operating mechanism, the thought of "dialogue" in literary criticism is mainly carried out from three logical levels: comparative proof-melting and complementing-innovation and transformation. In the context of dialogue, the birth of every thought or theory is a creative product that leaps forward between these three levels. They are viewed from the low level phenomenon, leap to the logic necessity of the high level, from the initial discourse mutual proof, complement each other, sublimate as the ultimate innovation and transformation of the theory, reflected the micro and macro, reflecting the microscopic and macro, phenomenological research paradigm and logical research paradigm of the perfect combination.

First of all, the first logical aspect of "dialogue" is mutual comparison. Starting from the details of the text and taking the conceptual category of literary theory as the basic standpoint, the critics extract a critical proposition that exists generally in the cross-era or heterogeneous cultural context. Then, by using critical methods such as "harmony", "contiguous category", "tracing the source along the wave", "catching a place", and so on, it breaks all kinds of boundary boundaries, such as ancient and modern, region, discipline, culture, etc. A number of relevant statements in different cultural contexts are "invited" to "please", so that these words of isomorphism or heteromorphism enter the dialogue with their vision of "insight" and "blind area", and show their strengths and weaknesses under the interactive reflection.

Secondly, the second logical aspect of the dialogue is melting complementarity. Comparison is not an end in itself. The meaning of dialogue lies in the exchange of ideas and the melting of the agreement. In particular, it affirms the cultural differences and their values in the oppositional context, and makes them complement each other in the academic system. On this level, critics criticize such critical methods as "comparison", "shenyin", "discrimination", "phase-to-talk", "letterhead", etc. On the basis of the preliminary "comparison" on the previous level, further introduce the discourse subject of the ancient and modern Chinese 
and Western discourse to the "fusion" dialogue, so as to achieve a thorough "visual integration" between each other, to enhance understanding, complement each other, eliminate the dregs, and improve themselves. As Hegel put it, "the understanding of customary things has increased, from 'knowing' (bekannt) to 'understanding' (erkannt), from old acquaintance to true acquaintance" (Qian, 2002a: p. 35).

Finally, the third logical level of dialogue is innovation and transformation. The purpose of dialogue is not only to melt complementarity, but its ultimate aim is to sublimate and innovate theory. Through the mutual proof and complementation of the first two levels, a common critical proposition, which is the theme of "dialogue", has completed the "cycle of interpretation" at all levels of the text, as well as between different disciplines, different times and different cultures. Under the multi-angle and multi-view circle view, it shows its abundant connotation and tension. Therefore, on this basis, the critics with unique insight and profound knowledge, theoretical innovation of the integration and transformation, in the new horizon is to complete the transformation and sublimation of the theory, and finally obtain different common wisdom. This "new view" of literature, which is based on the existing theoretical discourse, is not only the activation and creation of the conceptual category of Chinese traditional literary criticism, but also the promotion and enrichment of the western modern literary criticism thought. The new transcendence enriches the theory of Chinese and Western literary criticism.

The above three logical levels of mutual evidence, melting and complementation, innovation and transformation cannot be viewed in isolation or mechanically. The purpose of this division is to facilitate the understanding of the formation mechanism of dialogue. In fact, these three levels cannot be separated completely, they always melt together, together construct as the combination of dialogue and communication mechanism, together breed the profound and penetrating literary knowledge.

\section{Conclusion}

On the whole, the mechanism of dialogue is quite similar to that of horizon fusion put forward by modern hermeneutics guru Gadamer. The so-called "horizon fusion" means that in the process of interpretation, the visual field of the explanatory subject and the existing visual field of the object of interpretation are integrated together to form a large field of vision. Thus, the gap between history and reality, tradition and contemporary is overcame in the fusion of these two perspectives, to produce a new understanding beyond the original preconceptions of the interpreter and the original content of the works. This is similar to the theoretical realm of "complementary fit" pursued by "dialogue". The difference is that the "horizon fusion" is only an effective means to understand the text, and it is only the reconstruction of the meaning between the interpreter and the text. "Dialogue" emphasizes the "horizon fusion" between different texts, 
subjects and cultures. It is a more open theoretical consciousness with a wider vision and a wider content.

The neo-platonic master Plotinus divided the process of exploring truth into three stages: home, going out, and going home. This is similar to the situation in which Taoists and Zen schools use "home" as a metaphor whenever they come to the conclusion of speculation or the pursuit of the mind (Qian, 2002b: p. 82). Of course, this kind of heart or thought of "home", not simply repeat the cycle, but to achieve a higher degree of understanding leap, adding new content, new discoveries. It can be said that "dialogue" thinking is this kind of thought's journey of "home-out-home", which is a critical paradigm and realm of perfection.

\section{Conflicts of Interest}

The authors declare no conflicts of interest regarding the publication of this paper.

\section{References}

Qian, Z. S. (2002a). Seven Episodes. Beijing: Joint Publishing.

Qian, Z. S. (2002b). Writing on the Side of Life. Beijing: Joint Publishing.

Ye, X. S. (1986). Socrates and His Philosophy. Beijing: People's Publishing House. 